\title{
Fenêtres de détection des xénobiotiques dans le sang, les urines, la salive et les cheveux
}

\section{Detection times of drugs in blood, urine, oral fluid and hair}

Alain G. VERSTRAETE

\section{$R E ́ S U M E ́$}

Les données sur les fenêtres de détection sont le plus souvent basées sur des études d'administration contrôlée à des volontaires ou sur des études qui ont analysé les liquides biologiques de sujets qui arrêtent leur consommation (souvent chronique), par exemple en prison ou en cure de désintoxication.

Les durées de détection dépendent surtout de la dose et de la sensibilité de la méthode, mais aussi de la voie d'administration, de la durée de l'usage (aigu ou chronique), du choix du liquide biologique à analyser, de la nature de la molécule ou du métabolite recherché, du pH et de la concentration de l'urine et des variations inter-individuelles de métabolisation. En général, les durées de détection sont les plus longues dans les cheveux, suivies des urines, de la sueur, de la salive et du sang.

Après soumission chimique, si l'on utilise des techniques très sensibles, les substances peuvent être retrouvées dans le sang pendant 6 heures à 2 jours, dans les urines pendant 12 heures à 5 jours et dans la salive pendant 6 heures à 2 jours.

\section{MOTS-CLÉS}

Durée de détection, urine, sang, salive.

\section{SUMMARY}

Data on the windows of detection are based on studies of controlled administration of drugs to volunteers or on the analysis of biological samples of subjects who are forced to stop their (often chronic) use of drugs, e.g. because of imprisonment or detoxification.

The detection times depend mainly on the dose and sensitivity of the method used, but also on the route of administration, the duration of use (acute or chronic), the biological fluid that is analysed, the molecule or metabolite that is looked for, the $\mathrm{pH}$ and concentration of the urine and the interindividual variation in metabolism. In general the duration of detection is longest in hair followed by urine, sweat, saliva and blood.

If one uses sensitive techniques, after drug facilitated sexual assault, the substances can be found in blood for 6 hours to 2 days, in urine during 12 hours to 5 days, and in oral fluid from 6 hours to 2 days.

\section{KEY-WORDS}

Window of detection, urine, blood, oral fluid. 


\section{Introduction}

'La fenêtre de détection d'un xénobiotique est une "caractéristique très importante, et souvent il est demandé au toxicologue pendant combien de temps un produit peut être détecté après qu'il ait été administré. Il est difficile de donner une réponse à cette question, car la fenêtre de détection dépend de beaucoup de facteurs et relativement peu d'études ont été publiées sur le sujet. En effet, ces travaux sont difficiles à mener, car il s'agit d'administrer des produits (souvent illicites) à des volontaires sains. Il existe des études qui mesurent la fenêtre de détection de drogues après arrêt complet de la consommation, par exemple chez des sujets emprisonnés ou admis en cure de désintoxication. Si ces études donnent des renseignements précieux sur les temps de détection après usage de doses souvent élevées, elles ne donnent pas de renseignements sur la durée de détection après une dose unique, comme c'est le cas pour la soumission chimique.

Nous allons revoir plus en détail les durées de détection de l'amphétamine, de la methylènedioxyméthamphétamine (MDMA), du cannabis, de la cocaïne, des opiacés, des benzodiazépines, de l'acide gamma-hydroxybutyrique (GHB) et de l'alcool. Ces produits sont souvent utilisés dans les cas de soumission chimique $(1,2$, 3). Les durées de détection dans les urines (4) et la salive (5) ont été revues récemment.

\section{Facteurs qui influencent la fenêtre de détection}

Les durées de détection dépendent de nombreux facteurs : la dose, la voie d'administration, l'usage aigu ou chronique, le choix du liquide biologique, le seuil de détection de la technique analytique utilisée, la nature de la molécule ou du métabolite recherché, le $\mathrm{pH}$ et la concentration de l'urine et les variations inter-individuelles de métabolisation (4).

\section{Fenêtre de détection des différents produits}

\section{Amphétamine}

La dose habituelle d'amphétamine est de 10 à $30 \mathrm{mg}$, mais les sujets tolérants peuvent ingérer jusqu'à $2000 \mathrm{mg} /$ jour. Le temps de demi-vie est très variable en fonction du $\mathrm{pH}$ urinaire et va de 7 à 34 heures. Dans le sang, l'amphétamine est détectable (limite de détection (LDD) $4 \mathrm{ng} / \mathrm{mL}$ ) pendant 46 heures après l'ingestion d'une dose de $10 \mathrm{mg}(6)$.
Si le $\mathrm{pH}$ de l'urine est normal, environ $30 \%$ d'une dose d'amphétamine sera éliminée de façon inchangée, mais si le pH est de 5, 74\% est éliminé de forme inchangée (7). La fenêtre de détection dans les urines après une prise unique est de 1 à 3 jours. Dans une étude chez des prisonniers, la durée de détection était en moyenne de 4.8 jours après le début de l'incarcération, la durée maximale étant de près de 9 jours (8).

Dans la salive, l'amphétamine peut être détectée pendant 20 à 50 heures (LDD $10 \mathrm{ng} / \mathrm{mL}$ ) (9).

\section{Méthylènedioxyméthamphétamine (MDMA, ecstasy) et dérivés}

La dose habituelle varie entre 50 et $150 \mathrm{mg}$. Le temps de demi-vie est d'environ 7 à 8 heures. Dans une étude chez deux volontaires, l'administration de $100 \mathrm{mg}$ de MDMA était détectable dans le sang pendant 24 heures et dans les urines pendant plus de 48 heures (10).

La fenêtre de détection généralement admise dans les urines est de 1 à 3 jours (6).

Pour la MDEA, la fenêtre de détection urinaire après administration de $140 \mathrm{mg}$ variait entre 1.4 et 2.6 jours (11). Après administration de $100 \mathrm{mg}$ de MBDB, la durée de détection était de 36 heures dans les urines (LDD $8 \mathrm{ng} / \mathrm{mL}$ ) et de $17 \mathrm{~h}$ dans la salive (LDD $2 \mathrm{ng} / \mathrm{mL}$ ) (12). Vingt-quatre heures après l'administration de $100 \mathrm{mg}$ de MDMA chez 8 sujets, la concentration était de 13.5 (déviation standard (DS) 18.6 ) ng/mL dans le sang et de 126.2 (DS 101.8) $\mathrm{ng} / \mathrm{mL}$ dans la salive (13).

\section{Cannabis}

La dose absorbée après avoir fumé un joint de cannabis est variable, entre 5 et $30 \mathrm{mg}$. La concentration plasmatique du tétrahydrocannabinol (THC) présente un pic très rapide (de l'ordre de 3 à $8 \mathrm{~min}$ ), puis la concentration de THC diminue rapidement (temps de demivie d'environ 30 minutes). Le 11-nor-9-carboxydelta9-tetrahydrocannabinol (THC-COOH) a un temps de demi-vie beaucoup plus long, de l'ordre de 20 à 57 heures chez les usagers occasionnels et 3 à 13 jours chez les usagers réguliers. Le THC est détectable (limite de détection (LDD) $1 \mathrm{ng} / \mathrm{mL}$ ) pendant environ 5 heures dans le plasma et 10 heures dans les urines (LDD $10 \mathrm{ng} / \mathrm{mL}$ ). Le THC-COOH peut être détecté pendant beaucoup plus longtemps et une durée de détection (LDD $5 \mathrm{ng} / \mathrm{mL}$ ) de 25 jours dans le sang a été rapportée (6). Une étude effectuée à Lübeck chez 52 volontaires admis en cure de désintoxication à montré que le THC-COOH (LDD $10 \mathrm{ng} / \mathrm{mL}$ ) restait détectable dans le sérum pendant 23.8 heures en moyenne, et 49 heures au maximum (14). 
Après avoir fumé un joint contenant $1.75 \%$ de THC, les urines étaient positives (EMIT $>20 \mathrm{ng} / \mathrm{mL}$ ) pendant 2.1 jours, après un joint de $3.5 \%$ de THC, la durée de détection était de 3.8 jours (15). Chez les usagers chroniques, le métabolite inactif (THC-COOH) peut être détecté pendant des semaines voire des mois (16). L'étude de Lübeck chez 52 volontaires admis en cure de désintoxication a montré que le THC-COOH (LDD $10 \mathrm{ng} / \mathrm{mL}$ ) restait détectable dans les urines pendant 4.9 jours en moyenne, et 18 jours au maximum (14). La durée de détection la plus longue rapportée est de 93 jours (17).

Niedbala et al. ont mesuré les concentrations salivaires du THC après administration orale ou fumée de marihuana. Les LDD étaient respectivement de $1 \mathrm{ng} / \mathrm{mL}$ et $0.5 \mathrm{ng} / \mathrm{mL}$ pour l'ELISA et la chromatographie en phase gazeuse couplée à la spectrométrie de masse en tandem (CG-SM-SM). Toutes les analyses étaient positives jusqu'à 15 heures (extrêmes : 1-24 par ELISA) et $13 \mathrm{~h}$ (1-24) par CG-SM-SM. Les fenêtres de détection (dernier échantillon positif) étaient de $31 \mathrm{~h}$ (1-72) par ELISA et 34h (1-72) par CG-SM-SM (18). Dans cette étude le $\mathrm{THC}-\mathrm{COOH}$ était positif dans les urines pendant $58 \mathrm{~h}(16-72 \mathrm{~h})$.

\section{Cocaïne}

La dose habituelle varie entre 20 et $100 \mathrm{mg}$. La durée de détection de la cocaïne dans le sang est de 4 à 6 heures, la durée de détection de la benzoylecgonine peut atteindre 48 heures (6). Dans le sang, chez des utilisateurs chroniques, la benzoylecgonine (LDD $25 \mathrm{ng} / \mathrm{mL}$ ) était détectable pendant 5.1 heures en moyenne (maximum : 7.2 heures) (14).

La benzoylecgonine, un des métabolites principaux de la cocaïne est positive dans les urines pendant un à deux jours après une administration intraveineuse de $20 \mathrm{mg}$ (19). Après une dose plus élevée $(1.5 \mathrm{mg} / \mathrm{kg})$ par voie intranasale, la fenêtre de détection est de deux à trois jours (20). Chez des usagers chroniques (jusqu'à 10 grammes par jour), la benzoylecgonine a été détectée 22 jours après l'arrêt de la consommation (21). Dans l'étude de Lübeck chez 52 drogués chroniques, la cocaïne (LDD $50 \mathrm{ng} / \mathrm{mL}$ ) était détectable pendant $6.8 \mathrm{~h}$ en moyenne ( $24.8 \mathrm{~h}$ au maximum), la benzoylecgonine (LDD $50 \mathrm{ng} / \mathrm{mL}$ ) pendant $47.4 \mathrm{~h}$ (4.9 jours au maximum) et l'ecgonine méthylester (LDD $50 \mathrm{ng} / \mathrm{mL}$ ) pendant $35 \mathrm{~h}$ (maximum : 7 jours) (14).

Dans la salive, on peut détecter la cocaïne pendant 5 à 12 heures après une dose unique (5). En utilisant des méthodes capables de détecter $1 \mathrm{ng} / \mathrm{mL}$, on peut également détecter la benzoylecgonine pendant 12 à 24 heures. Chez les utilisateurs chroniques, la fenêtre de détection peut atteindre 10 jours (LDD $0.5 \mathrm{ng} / \mathrm{mL}$ ).

\section{Opiacés : héroïne et morphine}

L'héroïne est le plus souvent injectée ou fumée. La dose utilisée au début est d'environ $10 \mathrm{mg}$, mais les sujets tolérants peuvent utiliser jusqu'à 1 ou 2 grammes. La durée de détection de la morphine dans le sang (LDD $1 \mathrm{ng} / \mathrm{mL}$ ) était de 20 heures après administration de 12 ou de $20 \mathrm{mg}$ d'héroïne par voie intraveineuse chez un sujet (22). Après avoir fumé $10.5 \mathrm{mg}$ d'héroïne, la durée de détection variait entre 22 minutes et 2 heures (22). Après injection intramusculaire de $20 \mathrm{mg}$ de morphine, elle était détectable (LDD $0.6 \mathrm{ng} / \mathrm{mL}$ ) pendant 24 heures dans le plasma (23). Après administration de $9 \mathrm{mg}$ d'hérö̈ne par voie intranasale, la morphine était détectable (LDD $1 \mathrm{ng} / \mathrm{mL}$ ) dans le sang pendant 12 heures (24). Dans le sang des utilisateurs chroniques, la morphine totale (LDD 25 $\mathrm{ng} / \mathrm{mL}$ ) était détectable pendant 29.2 heures en moyenne (maximum : 5 jours), la morphine libre (LDD 10 $\mathrm{ng} / \mathrm{mL}$ ) pendant $14.4 \mathrm{~h}$ (maximum : 5 jours) (14).

Après administration de 3,6 et $12 \mathrm{mg}$ d'héroöne par voie intraveineuse, la 6-acétylmorphine est détectable dans les urines pendant respectivement $2.3,2.6$ et 4.5 heures. La morphine totale (LDD $300 \mathrm{ng} / \mathrm{mL}$ ) est détectable pendant $18.5,24.8$ et 35.3 heures. Après une dose inférieure à $7 \mathrm{mg}$, la durée de détection pour la morphine totale (LDD $300 \mathrm{ng} / \mathrm{mL}$ ) était de 7.4 à 32 heures. Pour les doses entre 10 et $15 \mathrm{mg}$, la durée de détection était de 11 à 54 heures (25).

Dans l'étude de Lübeck, la 6-acétylmorphine (LDD 10 $\mathrm{ng} / \mathrm{mL}$ ) était détectable pendant $5 \mathrm{~h}$ en moyenne (maximum $34.5 \mathrm{~h}$ ) et la morphine totale (LDD $25 \mathrm{ng} / \mathrm{mL}$ ) pendant 4.95 jours (maximum : 11.3 jours) dans les urines (14).

Dans la salive, la 6-acétylmorphine est détectable (LDD $1 \mathrm{ng} / \mathrm{mL}$ ) pendant 0.5 à 8 heures et la morphine pendant 12 à 24 heures (5).

\section{Benzodiazépines}

Les fenêtres de détection des benzodiazépines sont très variables, car leurs caractéristiques pharmacocinétiques sont très différentes. Le tableau 1, établi par la commission Drug Facilitated Sexual Assault de la Society of Forensic Toxicologists résume quelques données. Récemment, différents auteurs ont utilisé des méthodes très sensibles pour améliorer la fenêtre de détection $\mathrm{du}$ flunitrazépam et du clonazépam. Après administration de $2 \mathrm{mg}$ de flunitrazépam à 10 sujets, et en utilisant une méthode CG-SM en mode ionisation chimique négative (LDD pour le 7-aminoflunitrazepam de $10 \mathrm{pg} / \mathrm{mL}$ ), Negrusz et al. ont pu détecter le 7-aminoflunitrazepam dans les urines pendant 14 jours chez 6 sujets, 21 jours chez 1 sujet et 28 jours chez 3 sujets (26). Kintz et al. ont administré $1 \mathrm{mg}$ et ont pu détecter le 7-aminofluni- 
trazepam pendant 48 heures par chromatographie liquide couplée à une barrette de diodes, $72 \mathrm{~h}$ par CG-SM à impact électronique, $96 \mathrm{~h}$ par chromatographie liquide couplée à la spectrométrie de masse, ou CG-SM en mode ionisation chimique négative, et 120 heures par CG-SM-SM à ionisation chimique négative (27). Dans cette étude, les méthodes immunochimiques n'ont jamais donné de résultat positif.

Samyn et al. ont détecté le flunitrazépam et le 7-aminoflunitrazépam dans la salive après administration de $1 \mathrm{mg}$ de Rohypnol@ ${ }^{\circ}$. La durée de détection (LDD 50 $\mathrm{pg} / \mathrm{mL}$ pour le flunitrazépam, $100 \mathrm{pg} / \mathrm{mL}$ pour le 7 aminoflunitrazépam) ne dépassait pas 6 heures (28).

\section{Acide gamma-hydroxybutyrique (GHB)}

Le GHB est éliminé très rapidement (temps de demivie de l'ordre de 20 minutes). Comme c'est un produit endogène, il faut utiliser des seuils de positivité pour différencier une administration du produit des concentrations physiologiques. Les seuils suivants ont été proposés: sang sur EDTA $5 \mu \mathrm{g} / \mathrm{mL}$, sang post mortem 50 $\mu \mathrm{g} / \mathrm{mL}$, urines $10 \mu \mathrm{g} / \mathrm{mL}$ et cheveux $2 \mathrm{ng} / \mathrm{mg}$ (27). Le GHB peut être détecté pendant environ $5 \mathrm{~h}$ dans le sang et la salive, moins de 12 heures dans les urines. Par contre, en posant un patch à la sueur chez un sujet, 12 heures ou 24 heures après l'administration de $20 \mathrm{mg} / \mathrm{kg}$ de $\mathrm{GHB}$, on peut encore détecter des concentrations supra-physiologiques $(27,29)$.

\section{Alcool}

L'alcool est éliminé rapidement, avec une vitesse d'élimination entre 0.1 et $0.2 \mathrm{~g} / \mathrm{L} / \mathrm{h}$ (30). Une dose de 12 grammes sera donc détectable dans le sang pendant environ 3 heures. Si l'on recherche l'éthylglucuronide,

Tableau I : Dose, durée d'action, temps de demi-vie et fenêtre de détection (sang et urines) de sept benzodiazépines (présentation d'Adam Negrusz au congrès de la Society of Forensic Toxicologists, Détroit, 2002). Les fenêtres de détection sont obtenues avec des techniques analytiques "classiques". Elles peuvent être allongées en utilisant des techniques plus sensibles. on pourra détecter la consommation de $12 \mathrm{~g}$ d'alcool pendant 21 heures (27).

\section{Doses minimales détec- tables dans les cheveux}

Pour les cheveux, le temps de détection dépend de la longueur (les cheveux poussent entre 0.8 et 1.3 $\mathrm{cm} / \mathrm{mois}$ ). La question la plus importante est de savoir si une administration unique d'un produit peut être détectée. Il existe relativement peu de données. Une dose unique de $2 \mathrm{mg}$ de flunitrazépam est détectable dans les cheveux (31). Une dose de $3 \mathrm{mg}$ de clonazépam a été détectée chez 6 sujets sur 10 semaines (32).

Pour le cannabis, la consommation d'un joint par semaine correspond à une concentration de 0.02 à $3 \mathrm{ng} / \mathrm{mg}$ dans les cheveux. La consommation d'un comprimé de MDMA correspond à une concentration d'environ 0.5 ng/mg dans les cheveux, mais chez certains sujets, le MDMA n'est pas détectable. Des expériences ont montré que la consommation de $0.6 \mathrm{mg} / \mathrm{kg}$ de cocaïne est détectable dans les cheveux (communication au workshop on hair analysis, Bordeaux, juin 2001).

\section{Conclusion}

Les fenêtres de détection des produits utilisés pour la soumission chimique sont très variables. Les chances de détecter les substances augmentent quand on utilise les méthodes les plus sensibles (SM en tandem, ionisation chimique négative), si on analyse le métabolite qui persiste le plus longtemps et si on a à disposition le liquide biologique qui permet un temps de détection le plus long possible. En général, les fenêtres de détection sont les plus longues dans les cheveux, suivies des urines, de le sueur, de la salive et du sang. Il reste encore beaucoup d'inconnues, comme le temps de détection de certains produits après une dose unique, la détectabilité d'une dose unique d'un produit par l'analyse des cheveux et d'autres études sont nécessaires.

\begin{tabular}{|c|c|c|c|cc|}
\hline Produit & $\begin{array}{c}\text { Dose } \\
\text { (mg) }\end{array}$ & $\begin{array}{c}\text { Durée d'action } \\
\text { (heures) }\end{array}$ & $\begin{array}{c}\text { Temps de } \\
\text { demi-vie (h) }\end{array}$ & \multicolumn{2}{|c|}{ Fane tre de détection } \\
Urines
\end{tabular}




\section{Références}

1. Kintz P. La soumission chimique. Aspects analytiques et expertises médico-légales. Annal. toxicol. analyt. 2001 ; $13: 120-1$

2. Questel, F., Diament-Berger O., Lagier G., Soumission médicamenteuse: analyse d'une série de résultats toxicologiques urinaires. Annal. toxicol. analyt. $2001 ; 13: 121-2$.

3. ElSohly, M.-A., Salamone, S.-J. Prevalence of drugs used in cases of alleged sexual assault. J. Anal. Toxicol. 1999; $23: 141-6$.

4. Vandevenne M., Vandenbussche H., Verstraete A. Detection time of drugs of abuse in urine. Acta Clin. Belg. $2000 ; 55$ : 323-33.

5. Samyn N., Verstraete A., van Haeren C., Kintz P. Analysis of drugs of abuse in saliva. Forensic Sci Review $1999 ; 11: 1-19$.

6. Iten P.-X. Fahren under Drogen-oder Medikamenteneinfluss. Zürich : Institut für Rechtsmedizin der Universität Zürich, 1994.

7. Ghysel M. Amphétamines et derivés. In: Kintz P. Toxicologie et pharmacologie médicolégales. Paris: Elsevier, 1998 : 465-541.

8. Smith-Kielland A., Skuterud B., Mørland J. Urinary excretion of amphetamine after termination of drug abuse. J. Anal. Toxicol. $1997 ; 21: 325-9$.

9. Wan S.-H., Matin S.-B., Azarnoff D.-L. Kinetics, salivary excretion of amphetamine isomers, and effect of urinary pH. Clin. Pharmacol. Ther. $1978 ; 23$ : 585-90.

10. Pacifici R., Farre M., Picchini S., Ortuno J., Roset., P.-N., Zuccaro P., Segura J., de la Torre R. Sweat testing of MDMA with the Drugwipe analytical device: a controlled study with two volunteers. J. Anal. Toxicol. 2001 ; $25: 144-6$.

11. Ensslin H.-K., Kovar K.-A., Maurer H.-H. Toxicological detection of designer drug 3,4-methylenedioxyethylamphetamine and its metabolites in urine by GC-MS and FPIA. J. Chromatogr. B Biomed. Appl. 1996 ; 683 : 189-97.

12. Kintz $P$. Excretion of $M B D B$ and $B D B$ in urine, saliva, and sweat following single oral administration. J. Anal. Toxicol. $1997 ; 21: 570-5$.

13. Navarro M., Pichini S., Farre M., Ortuno J., Roset P.-N., Segura J., de la Torre R. Usefulness of saliva for measurement of 3,4-methylenedioxymethamphetamine and its metabolites: correlation with plasma drug concentrations and effect of salivary pH. Clin. Chem. $2001 ; 47: 1788-95$.

14. Reiter A., Hake J., Meissner C., Rohwer J., Friedrich H.J., Oehmichen M. Time of drug elimination in chronic drug abusers. Case study of 52 patients in a "low-step" detoxification ward. Forensic Sci. Int. 2001 ; 119 : 248-53.

15. Huestis M.-A., Mitchell J.-M., Cone E.-J. Urinary excretion profiles of 11-nor-9-carboxy-delta9-tetrahydrocannabinol in humans after single smoked doses of marijuana. J. Anal. Toxicol. $1996 ; 20: 441-52$.

16. Smith-Kielland A., Skuterud B., Mørland J.-G. Urinary excretion of 11-nor-9-carboxy- $\triangle 9$ THC (THCCOOH) and cannabinoids in drug users. J. Anal. Toxicol. 1999; 23 : 323-32.

17. Lafolie P., Beck O., Bleenow G., Boréus L., Borg S., Elwin C.-E., Karlsson L., Odelius G., Hjemdahl P. Importance of creatinine analyses of urine when screening for abused drugs. Clin. Chem. $1991 ; 37: 1927-31$.
18. Niedbala R-S., Kardos K.-W., Fritch D.-F., Kardos S., Fries T., Waga J., Robb J., Cone E.-J. Detection of marijuana use by oral fluid and urine analysis following single-dose administration of smoked and oral marijuana. J. Anal. Toxicol. $2001 ; 25: 289-303$.

19. Cone E., Menchen S., Mitchell J, Validity testing of commercial urine cocaine metabolite assays: I. Assay detection times, individual excretion patterns, and kinetics after cocaine administration to humans. JFSCA 1989 ; $34: 15-31$.

20. Hamilton H., Wallace J., Christenson J. Cocaine and benzoylecgonine excretion in humans. JFSCA $1977 ; 22$ : 697-707.

21. Weiss R., Gawin F. Protracted elimination of cocaine metabolites in long-term, high-dose cocaine abusers. American J. Med. $1988 ; 85: 879-80$.

22. Jenkins A.-J., Keenan R.-M., Henningfield J.-E., Cone E.-J. Pharmacokinetics and pharmacodynamics of smoked heroin. J. Anal. Toxicol. 1994 ; $18: 317-30$.

23. Cone E.-J. Testing human hair for drugs of abuse. I. Individual dose and time profiles of morphine and codeine in plasma, saliva, urine, and beard compared to druginduced effects on pupils and behavior. J. Anal. Toxicol. $1990 ; 14: 1-7$.

24. Goldberger,B.-A., Darwin W.-D., Grant T.-M., Allen A.C., Caplan Y.-H., Cone E.-J. Measurement of heroin and its metabolites by isotope-dilution electron-impact mass spectrometry. Clin. Chem. $1993 ; 39: 670-5$.

25. Smith M.-L., Shimomura E.-T., Summers J., Paul B.-D., Jenkins A.-J., Darwin W.-D., Cone E.-J. Urinary excretion profiles for total morphine, free morphine, and 6acetylmorphine following smoked and intravenous heroin. J. Anal. Toxicol. 2001 ; 25 : 504-14.

26. Negrusz A., Moore C.-M., Stockham T.-L., Poiser K.-R., Kern J.-L., Palaparthy R, Le N.-L., Janicak P.-G., Levy N.-A. Elimination of 7-aminoflunitrazepam and flunitrazepam in urine after a single dose of Rohypnol. J. Forensic Sci. $2000 ; 45: 1031-40$.

27. Kintz P., Villain M., Cirimele V., Goullé J.-P., Ludes B. Usage criminel de substances psycho-actives: le problème de la durée de détection. Acta Clin. Belg. 2002; supplement $1: 24-30$.

28. Samyn N., De Boeck G., Cirimele V., Verstraete A., Kintz P. Detection of flunitrazepam and 7-aminoflunitrazepam in oral fluid after controlled administration of Rohypnol. J. Anal. Toxicol. $2002 ; 26: 211$-5.

29. Kintz P., Goullé J.-P., Cirimele V., Ludes B. Window of detection of gamma-hydroxybutyrate in blood and saliva. Clin. Chem. $2001 ; 47: 2033-4$.

30. Deveaux, M. L'alcool. In Mura, P., éditeur: Alcool, médicaments stupéfiants et conduite automobile. Elsevier, Paris, 1999.

31.Negrusz A., Moore C.-M., Hinkel K.-B., Stockham T.-L., Verma M., Strong M.-J., Janicak P.-G. Deposition of 7 aminoflunitrazepam and flunitrazepam in hair after a single dose of Rohypnol.. J. Forensic Sci. 2001; 46: 1143-51.

32. Negrusz A., Moore C.-M., Kern J.-L., Janicak P.-G., Strong M.-J., Levy N.-A. Quantitation of clonazepam and its major metabolite 7-aminoclonazepam in hair. $J$. Anal. Toxicol. 2000; 24: 614-20. 